\title{
EVALUATION OF PHYTOESTROGENS OF SOYBEAN IN FORM OF SEMI SOLID PHARMACEUTICAL PASTE AS A GRAFT MATERIAL IN BONY DEFECTS
}

\author{
Hassan Abdel-Ghany * and Mohammed Khashaba*
}

\begin{abstract}
Purpose: to evaluate the effectiveness of phytoestrogens of Soybeans in form of semi solid pharmaceutical paste as a graft material in bony defects.

Material and Methods: Twelve bony defects were created in the parietal bones of nine New Zealand white rabbits. Two groups were divided (Six defects each). One gm of the purified active fraction obtained from seeds of Glycine max L. (Soybean) Family Leguminosae, rich in isoflavones was prepared in form of a semi solid pharmaceutical paste and have been used as graft material for defects of study group. While other defects in control group were left empty. Defects and surrounding tissue were decalcified and routinely processed for Haematoxylin and Eosin staining. The area percent of newly formed bone was estimated using an image analyzer computer system. Comparison of the results of both groups was carried out. ANOVA and T-Test were used for statistical analysis.
\end{abstract}

Results: Regularly arranged, osteoid newly formed bone almost filled the surgical defect in study group. Scarce bone trabeculae and wide fibrovascular marrow spaces were observed in the control group. This difference was statistically significant $(\mathrm{P}<0.0001)$.

Conclusion: The results of this study suggest that phytoestrogens obtained from Soybeans seeds in form of semi solid pharmaceutical paste is an effective alternative graft material. Further studies are needed on extended time intervals.

\section{INTRODUCTION}

Bone is the second most transplanted tissue in the body with approximately 3.5 million bone graft procedures performed each year ${ }^{(1)}$. There are many cases in which bone grafts are needed in large quantity such as for reconstruction of large bone defects caused by trauma, tumors, infections, and congenital defects, and also in cases where the regeneration is compromised(osteoporosis, necrosis and atrophic non-unions). All these facts emphasize that, large bone defects are still a challenge for maxillofacial surgeons ${ }^{(1)}$.

\footnotetext{
* Lecturer of Oral and Maxillofacial Surgery, Faculty of Oral and Dental Medicine, Cairo University.
} 
Generally four elements are needed of bone grafts for bone regeneration: Osteoconduction, osteoinduction, osteointegration, and osteogenesis ${ }^{(2)}$. Osteoconduction is the ability to support bone growth on a surgical site, during which pores, channels, and blood-vessels are formed within bone. Osteoblasts from the margin of the defect that is being grafted utilize the bone graft material as a framework upon which to spread and generate new bone. Osteoinduction involves the stimulation of osteoprogenitor cells to differentiate into osteoblasts that then begin new bone formation. The most widely studied type of osteoinductive cell mediators are bone morphogenetic proteins (BMPs) ${ }^{(2)}$.

A bone graft material that is osteoconductive and osteoinductive will not only serve as a scaffold for currently existing osteoblasts but will also trigger the formation of new osteoblasts. Osteointegration is the direct contact of living bone to graft material ${ }^{(3)}$. Finally osteogenesis is the formation of new bone by osteoblasts within the graft material. There are three main types of bone grafts, autografts, allograftsand bone graft substitutes. The autograft is considered as the standard in bone grafts. The ilium is the most frequent donor site accounting for onethird of all cases ${ }^{(3)}$.

However, its use is limited by complications such as pain, additional operating time, infection, scarring, blood loss, and donor site morbidity. The major limitations of using autogenous grafting includes donor site morbidity, pain and infection ${ }^{3}$. Moreover, graft resorption posed a severe problem. This encouraged the introduction of a variety of bone substitutes to aid in bone grafting. This included allografts, alloplasts and xenografts ${ }^{(4)}$.

Bone remodeling is the function of the activity of 2 different cell lines. Osteoblasts, responsible for bone formation, respond to changes in the activity of osteoclasts, the bone resorbing cells. Many hormones, growth factors, and cytokines play a regulatory role in maintaining bone homeostasis by their effects on these 2 cell lines, and estrogen in particular is responsible for suppressing osteoclast activity and thereby preventing bone resorption $(5,6)$

During menopause a marked decrease in the levels of estrogen has been detected. This showed a direct relationship with bone loss and osteoporosis, introducing estrogen replacement therapy as a treatment preventing bone loss ${ }^{(7)}$. Treatment by using estrogen enhances osteoblast differentiation and bone formation ${ }^{(8)}$.This direct positive effect was detected on the osteoblastic activity. However, an inverse relationship was made upon osteoclast. This was detected in the form of a decrease in the action and differentiation of osteoclasts . Therefore, estrogen is considered as an important treatment for bone osteoporosis and in maintenance of bone metabolism. ${ }^{(9)}$

Phytoestrogens are plant-derived non-steroidal compounds that bind to estrogen receptors (ERs) and have estrogen-like activity. There are three classes of phytoestrogens: isoflavones, coumestans and lignans $\mathrm{s}^{(9)}$. They have played an important role in various medical conditions such as prevention and treatment of cardiovascular diseases, osteoporosis, diabetes and obesity, menopausal symptoms, renal diseases and various types of cancer ${ }^{(10,11)}$.

A number of observational and dietary intervention studies confirm the general findings from the in vitro effects of phytoestrogens on bone cells in culture. Observational or epidemiologic studies ${ }^{(12,13)}$ and dietary intervention studies ${ }^{(14-17)}$ have shown significant relationships between phytoestrogens and surrogate markers for bone turnover that are indirectly consistent with reduced bone turnover markers indicative of osteoblast and osteoclast activity that have been measured include urinary calcium, magnesium and phosphorous, hydroxyproline, and collagen cross-links, while serum measures have included bone-specific alkaline phosphatase, tartarate-resistant acid phosphatase, osteocalcin, insulin-like growth factor I (IGF-I), and interleukin $6^{(18)}$.

Various studies made by Kannoetal..$^{(\mathbf{1 9}, 20)}$ detected the effects of phytoestrogens and environmental 
estrogens on osteoblast differentiation. This was applied on MC3T3-E1 cells, a mouse calvaria osteoblast-like cell line. They increased alkaline phosphatase activity and enhanced bone mineralization in these cells. Many users of Chinese herbal medicine believe that puerarin has positive effects in the treatment of coronary heart disease. In recent years puerarin injection has been widely used to treat coronary heart disease and angina pectoris.

In a study by Wang and colleagues in $2006^{(21)}$, the assessment of the benefits and harms of puerarin injection for unstable angina was made. This was applied on 1240 patients for 20 days to bring the fact that there was a strong evidence to suggest that puerarin injection plus western drugs was a better treatment option than western drug alone. This was proved by the improvement of the frequency of anginal attacks, ECG and incidence of nitroglycerin needed.

Soybean (Glycine $\max$ L. Merrill) were first grown as a crop in China about 5000 years ago ${ }^{(\mathbf{1 2})}$ and have been widely consumed as folk medicines in China, India, Japan and Korea for hundreds of years. Its nutrients become basic for humans consumption, beyond its by-products, that offer great diversities of products to the food industry. Soybean oil is highly consumed world-wide and soy milk is often used as a milk substitute to people who have lactose intolerance. In addition soybean has phytoestrogens which can be used in as a hormonal replacement to the decrease in estrogen levels ${ }^{(13)}$. One important group of minor compounds present in soybean that has received considerable attention is a class of phytoestrogen called the isoflavones ${ }^{(\mathbf{1 4})}$.

\section{MATERIALS AND METHODS}

\section{Ia) Plant Material :}

Seeds of Glycine max L. (Soybean) Family Leguminosae were purchased from Local Market and authenticated in the Herbarium of Flora Department, Agricultural Museum, Giza, Egypt.
The seeds were air dried, powdered and reduced to mesh no. 36 and kept in tightly sealed containers for pharmaceutical study.

The extraction, separation and identification of the flavonoidal compounds were made according to phytochemical methods. The seeds were extracted successively in a continuous extraction apparatus (Soxhlet) till exhaustion with petroleum ether (40$60 \mathrm{C})$ then with aqueous methanol. The methanol extract was concentrated to a small volume by evaporation under reduced pressure at a temperature not exceeding $40 \mathrm{C}$, then subjected to a polymide column and eluted with water with the proportional increasing of methanol. The obtained fractions were separately chromatographed on a Sephadex LH-2O column using methanol. Glycosides were hydrolyzed to their aglycones and sugars. The isolated compounds were investigated by High Performance Liquid chromatography (HPLC), comparing with authentic references, under the following conditions of flow rate $1 / \mathrm{min}$; Agilent 1100 series (Waldborn, Germany), quaternary pump (G1311A), Thermostated Autosamples (G1329A), variable wave length detector (G1314A) and column : Zorbax 300SB C18 column (Agilent Technologies, USA). Injection was carried out at wavelength $280 \mathrm{~nm}$ for separation. The solvent system consisted of methanol with $0.1 \%$ formic acid (solvent A); acetonitrile with $0.1 \%$ of formic acid (solvent B). Qualitative investigation of the active fraction revealed the presence of the isoflavones: genistein, daidazein, glycitein ,biochromin and fromononectin.

\section{Ib) Preparation of plant active fraction:}

One gm of the purified active fraction, rich in isoflavones was prepared in form of a semi solid pharmaceutical paste.

\section{IIa) Experimental and control groups}

Twelve 5-10 mm full-thickness bone defects were created in the parietal bones of nine New 
Zealand White rabbits from an inbred colony. The rabbits were 5 months old (adult stage) and weighed $3.5-4.0 \mathrm{~kg}$. The handling of the animals and the experimental protocol were approved by the Committee on the Use of Live Animals in Teaching and Research, the University of Cairo.

The rabbits were divided into 2 groups. As a double blinded study, Study Group included 6 defects filled with phytoestrogens obtained from Soybeans seeds in form of semi solid pharmaceutical paste, and Control Group where other 6 defects were left empty. This gives a total of 12 defects in the study, 3 Rabbits in each group and each rabbit have 2 parietal defects.

\section{IIb) Surgical Procedure}

After induction of anesthetic, the site of surgery was sterilized using Betadine, followed by draping. Local anesthetic was injected using Articaine 4\% with epinephrine 1:200000. A midline incision was made using Bard Parker no. 15 in layered fashion till the bone is reached. The surgical procedure consisted of the creation of two 5 X10X1 mm fullthickness cranial defects, devoid of periosteum, using templates, in the parietal bones. The defects were produced using round stainless steel burs on adequate speed dental drill. Outlines of the defects were made initially by making holes of full thickness the parietal bone using a stainless steel wire template bent to the required size of the defect.

The holes were joined to complete the process. During the cutting of bone, copious amount of sterile saline was used for irrigation and to minimize thermal damage to the tissues. Depending on which groups in which the rabbit belonged, the defects were grafted with study material or left empty.

\section{IIc) Postoperative care}

All wounds were closed with interrupted 3/0 black silk sutures. No attempt was made to approximate the periosteum to prevent the barrier effect. Postoperatively, the rabbits were given oxytetracycline hydrochloride daily and buprenorphine hydrochloride for 3 days. Two weeks after surgery, the animals were killed with sodium pentobarbitone. Immediately upon death, defects and surrounding tissue were removed for histological preparation.

\section{IIIa) Specimen preparation for microscopic ex- amination}

Immediately after sacrifice, the parietal bone was carefully dissected, immediately fixed in $10 \%$ formalin, then decalcified in ethylenediaminetetraacetic acid (EDTA) for 4 weeks. After decalcification, tissue blocks were routinely processed and embedded in paraffin. Fix $\mu \mathrm{m}$ sections were perpendicular to the long axis, mounted on glass slides, deparaffinized, hydrated and stained with Hematoxylin and Eosin stain for histological evaluation and histomorphometric analysis.

\section{IIIb) Measuring of the area percent of newly formed bone}

The area percent of newly formed bone was estimated using Leica Quin 500 analyzer computer system, (Leica Microsystems, Switzerland). The cursor was used to outline the areas of newly formed bone trabeculae, which were then masked by a binary colour that could be measured by the computer. The image analyzer is calibrated automatically to convert the measurement units (pixels) produced by the image analyzer program into actual micrometer units. The area percent of newly-formed bone was estimated in 10 different fields in 5 successive regions of the defect in each group using magnification (x200). Mean values and standard deviation (SD) were calculated for each group.

\section{IV- Statistical analysis:}

The data obtained from computer image analysis were tabulated and statistically analyzed. Numerical data were explored for normality by checking the data distribution, calculating the mean and median values, evaluating histograms and normality curves and using Kolmogorov-Smirnov and Shapiro-Wilk tests. 
Data were presented by mean, standard deviation (SD). One way ANOVA was used for comparison between groups followed by Tukey post hock test for repeated measures was used for comparison between different regions followed by simple main effect with bonferrioni correction. The significance level was set at $\mathrm{P} \leq 0.05$. Statistical analysis was performed with IBM ${ }^{\circledR}$ SPSS ${ }^{\circledR}$ Statistics Version 20 for Windows.

\section{RESULTS}

\section{a) Study Group:}

Microscopic examination revealed that the deposition of osteoid newly formed bone trabeculae starting from the defect border and growing through the defect. The bone trabeculae were regularly arranged, separated by bone marrow spaces and surrounded by an osteoblastic layer. Lacunae containing osteocytes were detected within the trabeculae. The newly formed bone consisted mainly of woven bone with some more mature lamellar bone apparent. The evident merging of woven and mature bone trabecula indicated that the maturation process was in progress. No inflammatory cell infiltrate was present (Fig 1: 1A, 1B, and 1C)

\section{b) Control group}

Microscopic examination revealed that there was no bone regeneration in the center of the bony defect, although there was evidence of vascular, fibrous and fatty tissue growing in the site. Wide marrow areas separated the scarce newly formed bone trabeculae. This marrow comprised both haemopoietic cells and adipocytes (Fig 1: CA, CB, and CC)

\section{Histomorphometric analysis}

Study group showed higher value compared to control group with region 2,3,4,5 and T test showed significance difference at region 2,3,4. Control group ANOVA for repeated measures showed significance difference between regions and post hock test showed that difference between region 1 and other regions were statistically significant except with region 5 was not statistically significant. Difference between region 2 and other regions were statistically significant except with region 4 was not statistically significant. Study group ANOVA for repeated measures showed significance difference between regions and post hock test showed that difference between region 1 and other regions were statistically significant except with region 5 was not statistically significant. Difference between region 2 and other regions were statistically significant except with region 3 were not statistically significant (Table 1, Fig. 2).

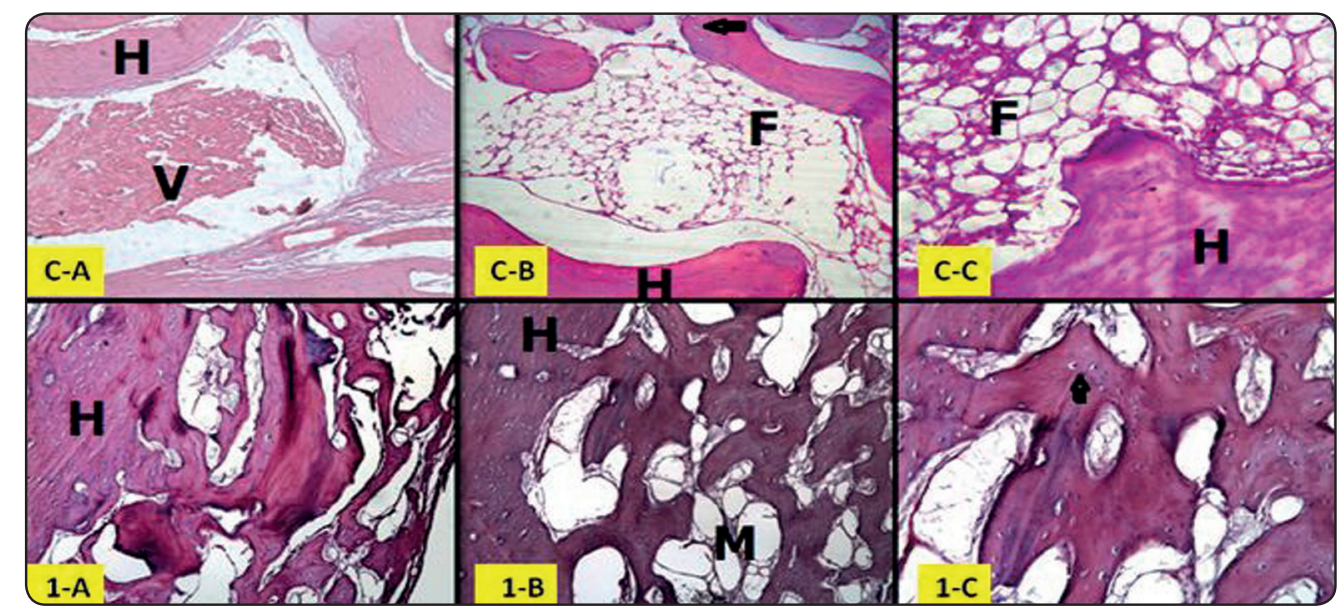

Fig. (1) Photomicrographs of both groups : 1- study group (1-A, 1-B, 1-C) showing interlacing bone trabeculae filling the defect area separated by fibro-vascular marrow $(\mathrm{M})$. Arrows point to viable osteocytes entrapped within bone, 2- control group (C-A, C-B, C-C) showing scarce bone formation separated by vacular (V) and fatty $(\mathrm{F})$ marrow. $(\mathrm{H})=$ host bone at the periphery of the defect, [(original magnification: H\&E x100] 
TABLE (1) Mean area \% occupied by bone trabeculae in different regions (where region 3 is the center of the defect) in both groups and significance of difference.

\begin{tabular}{|c|c|c|c|c|c|c|c|c|}
\hline & & $\begin{array}{c}\text { Region } \\
1\end{array}$ & $\begin{array}{c}\text { Region } \\
2\end{array}$ & $\begin{array}{c}\text { Region } 3 \\
\text { (center) }\end{array}$ & $\begin{array}{c}\text { Region } \\
4\end{array}$ & $\begin{array}{c}\text { Region } \\
5\end{array}$ & F value & $\mathrm{P}^{\#}$ value \\
\hline \multirow[t]{2}{*}{ Control } & Mean & $82.32^{\mathrm{a}}$ & $43.17^{b}$ & 21.6 & $39.87^{b}$ & $80.43^{a}$ & \multirow[t]{2}{*}{57.3} & \multirow[t]{2}{*}{$<0.0001$} \\
\hline & SD & 11.42 & 7.12 & 4.92 & 5.01 & 9.48 & & \\
\hline \multirow[t]{2}{*}{ Gp 1} & Mean & $80.19^{\mathrm{a}}$ & $65.72^{\mathrm{b}}$ & $58.78^{\mathrm{b}}$ & 70.68 & $81.80^{\mathrm{a}}$ & \multirow[t]{2}{*}{11.68} & \multirow[t]{2}{*}{$<0.0001$} \\
\hline & SD & 9.58 & 12.13 & 11.11 & 9.12 & 6.27 & & \\
\hline \multirow{2}{*}{$\begin{array}{c}\text { Significance of } \\
\text { difference between } \\
\text { groups }\end{array}$} & $\mathrm{t}$ value & 0.35 & 3.9271 & 7.4952 & 7.252 & 0.2953 & & \\
\hline & $P$ value & 0.7336 & 0.0028 & $<0.001$ & $<0.001$ & 0.7738 & & \\
\hline
\end{tabular}

Similar small superscript letters indicate no sig diff

*statistically significant, $n s=n o t$ significant, $P \#$ value= of difference between regions

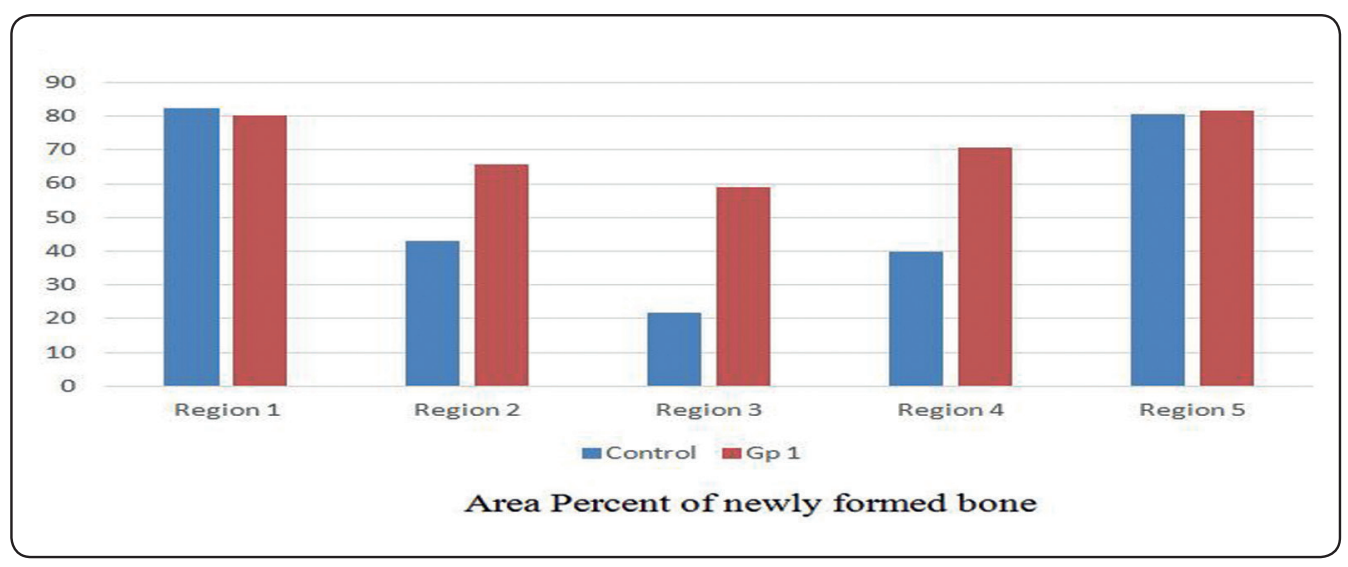

Fig. (2) Mean area percent occupied by newly formed bone in different regions of both groups.

\section{DISCUSSION}

Phytoestrogens are divided into three classes: isoflavones,coumestans and lignans. The most extensively studied phytoestrogens are isoflavones, which are found in high concentrations in soybeans. Phytoestrogens are often good antioxidants and antiinflamatory agents. These estrogen receptor (ER)independent properties of genistein, resveratrol and other isoflavones, indicate that they have the potential to affect a wide array of intracellular signaling mechanisms important for regulating cellular growth and protection. populations. A WHO Study Group Report states the incidence of osteoporosis is lower in Asian women than their Western counterparts .This is verified in an epidemiological review by Cooper and colleagues. A recent article by Anderson and colleagues reported that a low dose of genistein as equivalent to Premarin (WyethAyerst Laboratories) in maintaining bone mass in ovarectomised rats.

The promotion of bone healing observed after administration of soybean in the present work was attributed to the beneficial effects of isoflavones, which is in agreement with Alekel et al and Kaneki et $\boldsymbol{a l}^{(22,23)}$. These results are also consistent with Arjmandi and Smith, Dijsselbloem et al, and many other studies $^{(24-30)}$. 
Most of the published literature suggests that soybean exerts its bone-preserving properties via maintenance of high vitamin $\mathrm{K}$ status ${ }^{(24)}$, and the ability of soy protein to decrease bone dissolution and urinary calcium excretion ${ }^{(13)}$ as well as binding of isoflavones to estrogens ${ }^{(31-32)}$.

In the current study, rabbits were selected as experimental animals. The rabbit is one of the most commonly used animals for medical research, being used in approximately 35\% of musculoskeletal research studies. ${ }^{(33)}$ This is in part due to ease of handling and size, as well as there is minimal literature on the differences between human and rabbit bone composition and density ${ }^{(34)}$.Moreover, in comparison to other species, such as primates and some rodents, the rabbit has faster skeletal change and bone turnover ${ }^{(35-37)}$.

The results of this study showed an increase in bone formation after 14 days. This was assessed by histomorphometric analysis and bone area percent from the central part of the defect. It showed the higher bone formation in Study Group, filled with phytoestrogens obtained from Soybeans seeds in form of semi solid pharmaceutical paste, of a value of $58.78 \%$. Whereas the Control Group where bone defects were left empty, the results were only $21.6 \%$. The increase in bone formation in Study Group was $272 \%$ compared to Control Group. The area percent of newly formed bone was estimated using an image analyzer computer system. ANOVA test was used for statistical analysis. The difference between the groups was statistically significant $(\mathrm{P}<0.0001)$.

The effect of Isoflavones on the healing of bony defects experimentally was early tested by Wong and $\boldsymbol{R a b i e}^{(10)}$. It was applied in a similar study design but using Puerarin, an isoflavonoid isolated from a wild leguminous creeper called Kudzu. It resulted in an increase in bone formation by $554 \%$.

This study showed, that the semi solid pharmaceutical paste obtained from Soybeans seeds is an effective graft material. This can be attributed to the beneficial effects of isoflavones.

\section{CONCLUSION}

Phytoestrogens have the potential to improve bone healing in surgical defects. The semi solid pharmaceutical paste obtained from Soybeans seeds is an effective graft material. It is recommended that more studies be made using different timings for testing the fate of the graft material.

\section{ACKNOWLEDGEMENT}

We are thankful to our colleagues Prof. Nagwa M. Ammar, and Dr. Amira A. El Anssary who provided expertise that greatly assisted the research. We are also grateful to Prof. Dalia El Rouby for assistance with Histomorphometric analysis of the result of this study.

\section{REFERENCES}

1. Wang M.: Developing bioactive composite materials for tissue replacement. Biomaterials. 24:2133-2151, 2003.

2. Kheiralla M, Al Mashaly H: Bone Graft Substitutes for Bone Defect Regeneration. A Collective Review. Int J Dentistry Oral Sci. 03(5), 247-257,2016.

3. Misch CE, Dietsh F.: Bone - grafting materials in implant dentistry. Implant Dent. 2: 158-167, 1993.

4. Calori G.M, Mazza E. :The use of bone-graft substitutes in large bone defects: Any specific needs? Injury42: S56S63, 2011.

5. Stelzle F:.Clinical outcomes of sinus floor augmentation for implant placement using autogenous bone or bone substitutes: a systematic review.Clinical Oral Impl Research 20: 124-133, 2009.

6. Blair HC, Schlesinger PH, Ross FP, Teitelbaum SL. Recent advances toward understanding osteoclast physiology. ClinOrthop 1993; 294:7-22.

7. Manolagas SC, Jilka RL.Bone marrow, cytokines, and bone remodeling: emerging insights into the pathophysiology of osteoporosis. N Engl J Med1995; 332:305-11.

8. Voss PJ: Maxillary sinus augmentation with autogenous bone grafts to enable placement of SLA-surfaced implants: preliminary results after 15-40 months. Clin. Oral Implants Res 14:207-212, 2003. 
9. Turner RT: Skeletal Effects of Estrogen. Endocr Rev 1994; 15:275e300.

10. Wong R, Rabie B.: Effect of Puerarin on bone formation. Osteoarthritis and cartilage.15, 894-899, 2007

11. Garner S., Anderson J: The effects of Phytoestrogens on bone. Nutrition Research. 17(10): 1617-1632, 1997

12. Li B, Yu S: Effect of Puerarin on the bone metabolism in vitro. Beijing Da XueBao. 35(1): 74-77, 2003.

13. Messina, M.J.: Soy Foods and Soybean isoflavones and Menopausal Health. PhD NutrClin Care., 5: 272-282, 2002.

14. Horiuchi T, Onouchi T, Takahashi M, Ito H, Orimo H. Effect of soy protein on bone metabolism in postmenopausal women. Osteoporosis 2000; 11:721-4.

15. Fukui Y, Miura A, Nara Y, Uesugi T, Yamori H, Yamori Y. Relationship between urinary isoflavones and bone metabolism in postmenopausal Japanese women. J Nutr 2000;130:686S (abstr).

16. Lu L-JW, Anderson KE, Grady JJ, Nagamani M. Chronic soy consumption influences serum levels of steroid and peptide hormones without uterine effects in postmenopausal women. J Nutr 2002; 132:615S (abstr).

17. Teramoto T, Sakamoto A, Toda T, Okuhira T, Koketsu I. Effects of ingesting a beverage containing soy isoflavones on urinary bone resorption markers. J Nutr 2002; 132:614S (abstr).

18. Cook A, Pennington G. Phytoestrogen and multiple vita$\mathrm{min} /$ mineral effects on bone mineral density in early postmenopausal women: a pilot study. J Womens Health 2002; $11: 53-60$.

19. Setchell K and Lydeking-Olsen E. Dietary phytoestrogens and their effect on bone: evidence from in vitro and in vivo, human observational, and dietary intervention studies. Am J ClinNutr 2003; 78(suppl):593S-609S.

20. Kanno S, Ashby J, Jacob E.: Selecting the proper rodent diet for endocrine research and testing it metabolic effect on bone. Br J Nutr. 85, 307-316, 2004.

21. Kanno S, Hirano S, Kayama F.: Effects of phytoestrogens and environmental oestrogens on osteoblastic differentiation. Toxicology 196: 137-145, 2004.

22. Wang, Q., Wu, T. : Puerarin injection for unstable angina pectoris . Cochrane database Sys. Rev. 3, CD0004196.
23. Alekel, D.L.; St Germain, A.; Peterson, C.T.; Hanson, K.B.; Stewart, J.W. and Toda, T.: Isoflavone-rich soy protein isolate attenuates bone loss in the lumbar spine of perimenopausal women. Am J Clinical Nutr., 72(3):844-852, 2000 .

24. Kaneki, M.; Hedges, S.J.; Hosoi, T.; Fujiwara, S.; Lyons, A.; Crean, J.; Ishida, N.; Nakagwa, M.; Takechi, M.; Sano, Y.; Mizuno, Y.; Hoshino, S.; Miyao, M.; Inoue, S.; Horiki, K.; Shiraki, M.; Ouchi, Y. and Orimo, H.: Japanese fermented soybean food as the major determinant of the large geographic difference in circulating levels of vitamin K2: possible implication for hip fracture risk. Nutrition, 17: 315-321, 2001.

25. Arjmandi, B.H. and Smith, B.J.: Soy isoflavones' osteoprotective role in postmenopausal women: mechanism of action. J NutrBiochem., 13: 130-137, 2002.

26. Uesugi, T.; Fukui, Y. and Yamori, Y.: Beneficial effects of soybean isoflavone supplementation on bone metabolism and serum lipids in postmenopausal japanese women: a four-week study. J Am CollNutr., 21(2): 97-102, 2002.

27. Blum, S.C.; Heaton, S.N.; Bowman, B.M.; Hegsted, M. and Miller, S.C.: Dietary soy protein maintains some indices of bone mineral density and bone formation in aged ovariectomized rats. J Nutr., 133(5): 1244-1249, 2003.

28. Fonseca, D. and Ward, W.E.:Daidzein together with high calcium preserve bone mass and biomechanical strength at multiple sites in ovariectomized mice. Bone, 35: 489497, 2004.

29. Dang, Z.C. and Lowik, C.: Dose-dependent effects of phytoestrogens on bone. TRENDS EndocrinolMetabol., 16(5): 207-213, 2005.

30. Devareddy, L.; Khalil, D.A.; Smith, B.J.; Lucas, E.A.; Soung, D.Y.; Marlow, D.D. and Arjmandi, B.H.: Soy moderately improves microstructural properties without affecting bone mass in an ovariectomized rat model of osteoporosis. Bone, 38: 686-693, 2006.

31. Dijsselbloem, N.; Berghe, W.V.; De Naeyer, A. and Haegeman, G.: Soy isoflavonephyto-pharmaceuticals in interleukin-6 affections multi-purpose nutraceuticals at the crossroad of hormone replacement, anti-cancer and antiinflammatory therapy. BiochemPharmacol., 68: 1171$1185,2004$.

32. Y-X. Zhou et al. :Puerarin: A review of Pharmacological effects. Phytother. Res(2013) 
33. Fitzpatrick LA:Phytoestrogens - mechanism of action and effect of bone markers and bone mineral density. EndocrinolMetabClin North Am. 32(1):233-52, viii, 2003.

34. Neyt JG, Buckwalter JA, Carroll NC (1998) Use of animal models in musculoskeletal research. Iowa Orthop J 18: 118-123.

35. Pearce AI, RG Richards, S Milz, E Schneider and SG Pearce. Animal models for implant biomaterial research in bone: A review. European Cells and Materials Vol. 13: $1-10,2007$.

36. Castaneda S, Largo R, Calvo E, Rodriguez-Salvanes F, Marcos ME, Diaz-Curiel M, Herrero-Beaumont G Bone mineral measurements of subchondral and trabecular bone in healthy and osteoporotic rabbits. Skeletal Radiol 35:34-41, 2006.

37. Newman E, Turner AS, Wark JD. The potential of sheep for the study of osteopenia: current status and comparison with other animal models. Bone 16: 277S-284S, 1995. 